\title{
DNP measurements of irradiated deuterated polyethylene
}

\author{
Li Wang ${ }^{* a b}$

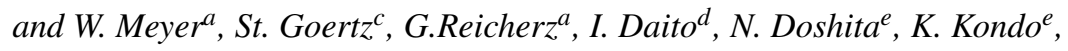 \\ T. Iwata ${ }^{e}$, N. Horikawa ${ }^{f}$ \\ ${ }^{a}$ Institut für Experimentalpysik I, Ruhr-Universität Bochum, 44780 Bochum, Germany \\ ${ }^{b}$ Physics Department, School of Science, Donghua University, Shanghai 200051, China \\ ${ }^{c}$ Physics Institute, University Bonn, D-53115 Bonn, Germany \\ ${ }^{d}$ Advanced Photon Research Center, Japan Atomic Energy Research Institute (JAERI Kansai), \\ Japan \\ ${ }^{e}$ Physics Department, Faculty of Science, Yamagata University, Yamagata, 990-8560,Japan \\ ${ }^{f}$ College of Engineering Chubu University, Kasugai, 487-850, Japan \\ E-mail: wang_li@dhu.edu.cn, meyer@epl.rub.de
}

Deuterated polyethylene samples have been prepared as polarizable target material. The paramagnetic centers necessary for the dynamic nuclear polarization process have been created by irradiation with electrons. The paramagnetic resonance from the induced centers into the samples has been studied in a conventional X-band EPR spectrometer operating at $9.35 \mathrm{GHz}$ and at liquid nitrogen temperature. In a fully deuterated polyethylene sample more than $30 \%$ deuteron polarization has been achieved at dilution refrigerator temperature and $2.5 \mathrm{~T}$.

XVIth International Workshop in Polarized Sources, Targets, and Polarimetry, PSTP2015,

14-18 September 2015,

Bochum, Germany

${ }^{*}$ Speaker. 


\section{Introduction}

Since the early 1960's polarized solid targets using the DNP (Dynamic Nuclear Polarization) process to enhance the proton or deuteron polarization are used [1]. Paramagnetic centers are necessary to transfer the high electron polarization obtained at a high magnetic field $(>2.5 \mathrm{~T})$ and at low temperatures $(<1 \mathrm{~K})$ to the nuclei. There are two generally applicable methods to induce paramagnetic centers into the target materials, (1) chemically doping (2) to irradiate the material such as ammonia, deuterated ammonia or deuterated lithium by ionizing radiation, e.g. by an intense electron beam [2, 3, 4]. For an effective DNP process, a number of $2 \times 10^{19} \mathrm{spins} / \mathrm{g}$ were shown to be most suitable.

The advantage of polymers as target materials, such as polyethylene or polystyrene, is the possibility to form them into different shapes and thicknesses at room temperature to fulfill the needs of particle physics experiments. This is especially beneficial for scattering experiments at low energies, where specially shaped thin targets are required.

Deuterated polyethylene foil targets with a thickness of 20 - $30 \mu \mathrm{m}$ have been used in a nuclear fusion experiments with a $20 \mathrm{MeV}$ polarized deuteron beam. Deuteron polarization values of about $10 \%$ [5] have been measured at $2.5 \mathrm{~T}$ and $0.8 \mathrm{~K}$. TEMPO radicals, introduced into the target foils by a heating procedure, have been used as paramagnetic centers [6].

Up to now, the highest deuteron polarization of polymer materials are as follows: (a) Irradiated polyethylene: Deuteron polarization values close to $30 \%$ have been obtained at a temperature of $1 \mathrm{~K}$ and $5 \mathrm{~T}[7,8]$. (b) Chemically doped polyethylene: TEMPO doped foil with deuteron polarization of $10 \%$ [5]. (c) Chemically doped polystyrene: In a Finland D36 doped deuterated polystyrene foil sample $61.5 \%$ deuteron polarization has been obtained in a dilution refrigerator at $5.0 \mathrm{~T}[9]$.

Here we report on DNP results of various samples of deuterated polyethylene after irradiation at the dose in the range between $1.0 \times 10^{14}$ and $1.0 \times 10^{17} e^{-} / \mathrm{cm}^{2}$. All deuteron polarization were obtained at $2.5 \mathrm{~T}$ in a dilution refrigerator. All EPR spectra have been studied by a X-band spectrometer at liquid nitrogen temperature.

\section{Material preparation for the DNP process}

Polyethylene samples were prepared starting with powder (Japan Isotope), warming and pressing it at $160^{\circ} \mathrm{C}$ for two hours and cooling down to room temperature. Such produced polyethylene foil samples have been irradiated at the injection Linac of the Frontier Science Innovation Center of the Osaka University, which provides $7 \mathrm{MeV}$ electrons with a time-averaged beam current of 25 $\mu \mathrm{A}$. The samples were kept under liquid nitrogen at $77 \mathrm{~K}$ during the irradiation process. The irradiation dose for different samples varied between $3.0 \times 10^{14}-1.0 \times 10^{17} e^{-} / \mathrm{cm}^{2}$. Other deuterated polyethylene samples have been irradiated at the Bonn injection Linac, which provides $20 \mathrm{MeV}$ electrons with a time-averaged beam current of $10-20 \mu \mathrm{A}$. The irradiation procedure at the Bonn Linac is done at $90 \mathrm{~K}$, where the samples are kept under liquid argon in a special set-up [10]. In the Table 1 the different samples with their spin density of the paramagnetic radicals are listed. The spin density has been determined by comparing the integrated EPR-line intensity with such of chemically doped samples of known radical concentration. 


\begin{tabular}{ccccc}
\hline Sample & {$\left[\mathrm{e}^{-} / \mathrm{cm}^{2}\right]$} & Spin density $\left[\mathrm{e}^{-} / \mathrm{g}\right]$ & Irradiation temp. $[\mathrm{K}]$ & Irr. facility \\
\hline $\mathrm{a}$ & $3.0 \times 10^{14}$ & $1.8 \times 10^{18}$ & 77 & Osaka \\
$\mathrm{b}$ & $7.0 \times 10^{14}$ & $2.7 \times 10^{18}$ & 77 & Osaka \\
$\mathrm{c}$ & $4.0 \times 10^{15}$ & $1.9 \times 10^{19}$ & 77 & Bonn \\
$\mathrm{d}$ & $6.0 \times 10^{15}$ & $2.3 \times 10^{19}$ & 90 & Bonn \\
$\mathrm{e}$ & $8.0 \times 10^{15}$ & $3.2 \times 10^{19}$ & 77 & Bonn \\
$\mathrm{f}$ & $1.0 \times 10^{16}$ & $3.6 \times 10^{19}$ & 77 & Osaka \\
$\mathrm{g}$ & $1.2 \times 10^{16}$ & $4.0 \times 10^{19}$ & 90 & Bonn \\
$\mathrm{h}$ & $5.0 \times 10^{16}$ & $2.3 \times 10^{20}$ & 77 & Osaka \\
$\mathrm{i}$ & $1.0 \times 10^{17}$ & $1.4 \times 10^{21}$ & 77 & Osaka \\
\hline
\end{tabular}

Table 1: Irradiation dose and irradiation temperature of deuterated polyethylene samples

\section{The nature of the irradiation-induced defects in deuterated polyethylene}

In order to better understand the influence of the properties of paramagnetic centers on the efficiency of the DNP process, the structure of the paramagnetic defects induced by irradiation should be determined.

Figure 1 shows the EPR line of deuterated polyethylene taken at liquid nitrogen temperature. A clear structure composed of eleven nearly equidistant transitions is observed. The characteristic

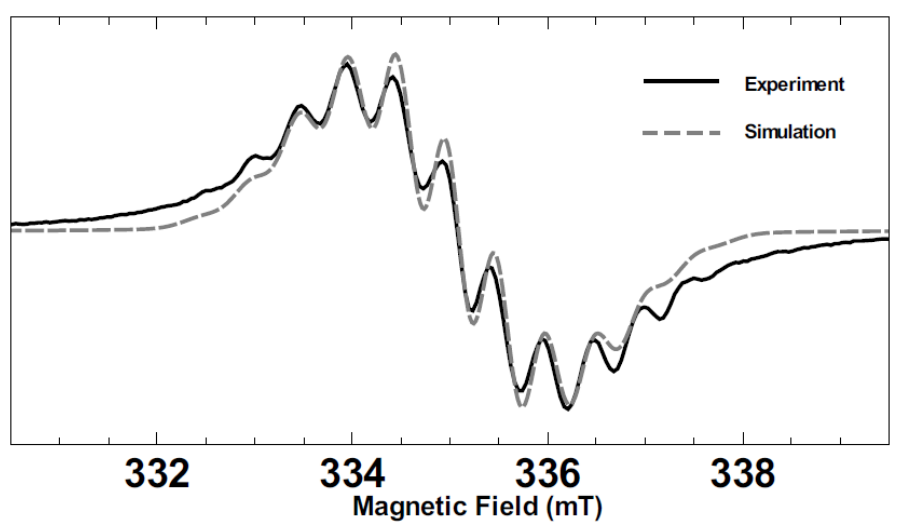

Figure 1: X-band EPR spectrum of electron-irradiated deuterated polyethylene at liquid nitrogen temperature

structure is in agreement with the radical position shown in Figure 2. The hyperfine structure corresponds to the residual unpaired electron and its wave function spreads over the respective carbon atom and the 5 adjacent deuterons, one deuteron on an $\alpha$-site (see Figure 2) and four deuterons on the $\beta$-sites of the carbon [11].

If the wave function spreads over $n$ equivalent spin- 1 particles, one expects $2 n+1$ hyperfine lines, in our case $2 n+1=11$. These lines can be easily identified within Figure 1 and are thus suggesting the defect picture shown above.

The simulated spectrum in Figure 1 was obtained by the simulation software made by JEOL, version 2.20. The EPR spectral parameters from a simulation are as follows: $g=2.0030$, H=hyperfine 


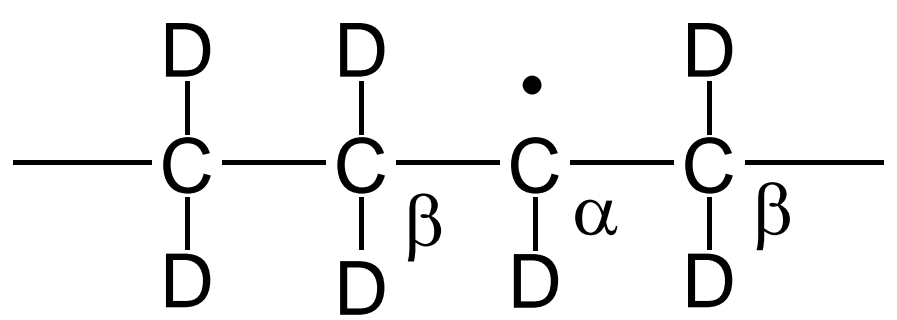

Figure 2: Radicals after irradiation of deuterated polyethylene

constant, $\mathrm{H}_{\alpha}=0.485 \mathrm{mT}(1 \mathrm{D}), \mathrm{H}_{\beta}=0.480 \mathrm{mT}(4 \mathrm{D})$; the linewidth (FWHM of the integrated line) FWHM $=3.0 \pm 0.2 \mathrm{mT}$. Given the symmetry of the EPR lines, one suggests only a small contribution from a possible anisotropy of the g-factor of these centers. The major contribution to the line broadening results from hyperfine interaction. Since the strength of this interaction is independent of the magnetic field, it is assumed that the EPR line of these materials will be of a similar width at the high magnetic field values, under which the dynamic nuclear polarization takes place.

\section{Polarization results of deuterated polyethylene samples}

The polarization measurements have been performed at a magnetic field of $2.5 \mathrm{~T}$ and at different temperatures between $130 \mathrm{mK}$ and $540 \mathrm{mK}$ using IMPATT-diodes microwave frequency generators (70 GHz at $140 \mathrm{~mW}$ ). Table 2 shows polarization values, irradiation doses, the applied microwave frequencies and the polarization build-up time. The polarization values were calculated by fitting the area and asymmetry method. Statistical errors are about $\pm 4 \%$ for the higher polarizations and $\pm 10 \%$ for the low polarization values. Fig. 3 shows a build-up curve of the polarization. The build-up time to reach $70 \%$ of the final polarization is about 100 minutes.

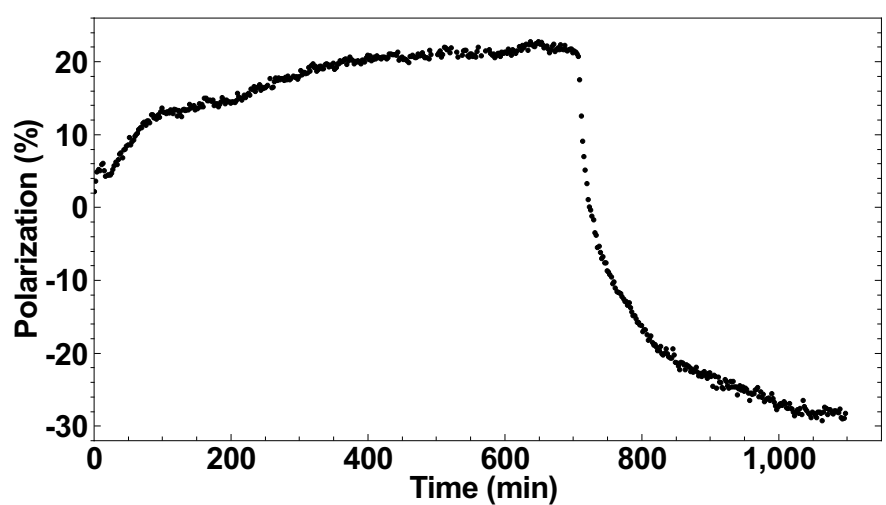

Figure 3: Build-up curve of the polarization in deuterated polyethylene at $150 \mathrm{mK}$ and $2.5 \mathrm{~T}$

Some comments to Table 2 are as follows: (a) As expected the best deuteron polarization results have been obtained at the lowest available temperatures, i.e. samples d, e and f. They were irradiated with doses from 6.0 to $12.0 \times 10^{15} e^{-} / \mathrm{cm}^{2}$. (b) The frequency difference $\left(\mathrm{f}^{+}-\right.$ $\mathrm{f}^{-}$) increases with increasing irradiation doses, which affects the strength of dipolar interaction 


\begin{tabular}{|c|c|c|c|c|c|c|}
\hline Sample & Dose $\left[\mathrm{e}^{-} / \mathrm{cm}^{2}\right]$ & DNP Temp.[mK] & $\mathrm{f}_{m w}[\mathrm{GHz}]$ & d-Pol[\%] & $\mathrm{T}_{\text {build-up }}$ [min.] & $\mathrm{f}^{+}-\mathrm{f}^{-}[\mathrm{MHz}]$ \\
\hline $\mathrm{a}$ & $3.0 \times 10^{14}$ & 530 & 69.970 & +3.7 & - & - \\
\hline $\mathrm{b}$ & $7.0 \times 10^{14}$ & 540 & 69.970 & +1.4 & - & - \\
\hline \multirow[t]{2}{*}{$\mathrm{c}$} & $4.0 \times 10^{15}$ & 130 & 69.876 & +22.8 & 85 & 194 \\
\hline & & & 70.070 & -28.9 & & \\
\hline \multirow[t]{2}{*}{ d } & $6.0 \times 10^{15}$ & 150 & 69.860 & +21.0 & 110 & 215 \\
\hline & & & 70.075 & -31.0 & & \\
\hline \multirow[t]{2}{*}{$\mathrm{e}$} & $8.0 \times 10^{15}$ & 140 & 69.890 & +19.1 & 100 & 240 \\
\hline & & & 70.150 & -31.1 & & \\
\hline \multirow[t]{2}{*}{$\mathrm{f}$} & $1.0 \times 10^{16}$ & 480 & 69.880 & +16.7 & 95 & 250 \\
\hline & & & 70.130 & -15.4 & & \\
\hline \multirow[t]{2}{*}{ g } & $1.2 \times 10^{16}$ & 150 & 69.880 & +18.7 & 83 & 270 \\
\hline & & & 70.150 & -31.1 & & \\
\hline \multirow[t]{2}{*}{$\mathrm{h}$} & $5.0 \times 10^{16}$ & 350 & 69.470 & +5.8 & - & - \\
\hline & & & 69.880 & -2.1 & & \\
\hline \multirow[t]{2}{*}{$\mathrm{i}$} & $1.0 \times 10^{17}$ & 350 & 69.420 & +1.6 & - & - \\
\hline & & & 69.950 & -1.9 & & \\
\hline
\end{tabular}

Table 2: Polarization results of deuterated polyethylene at $2.5 \mathrm{~T}$

between the radicals. The optimum frequency difference for the highest polarization is around 240 $\mathrm{MHz}$, which is smaller compared to TEMPO-doped deuterated polyethylene (340 MHz) [6]. (c) Irradiated deuterated polyethylene did not polarize quite well at an irradiation dose in the range of $10^{14} e^{-} / \mathrm{cm}^{2}$ and $10^{17} e^{-} / \mathrm{cm}^{2}$.

Due to the small magnetic moment of the deuteron and the finite EPR width of paramagnetic centers introduced in a solid-state material, a deuteron spin system is always in thermal contact to the electron non-Zeeman reservoir (describing the interaction among the electrons). Thus it is the equal spin temperature effect, which provides the relevant polarization mechanism in this case. This is manifested by the frequencies distance, at which optimum positive and negative polarization values have been measured.

\section{Summary}

The highest deuteron polarization of more than $30 \%$ can be achieved at the optimum irradiation dose in the range of $6-8 \times 10^{15} \mathrm{e}^{-} / \mathrm{cm}^{2}$. This dose is more than one order of magnitude less compared to the doses usually given to ammonia or ${ }^{6} \mathrm{LiD}$ samples. Therefore the irradiation procedure at the above mentioned intensities can be performed in the order of minutes. However, the big difference in the maximum positive and negative polarization values as measured is not yet understood.

In Table 3 the highest deuteron polarization values of deuterated polyethylene, measured by different polarized target groups, are summarized. Taking into account the slightly higher dilution 


\begin{tabular}{cccccc}
\hline Material & $\mathrm{f}_{\text {dilution }}$ & Radical & DNP condition & $\left|\mathrm{P}_{d, \max }\right|$ & Laboratory \\
\hline $\mathrm{CD}_{2}$ & 0.25 & $\mathrm{TEMPO}$ & $1.1 \mathrm{~K} / 2.5 \mathrm{~T}$ & $10 \%$ & PSI [6] \\
$\mathrm{CD}_{2}$ & 0.25 & $\mathrm{CD}$ & $150 \mathrm{mK} / 2.5 \mathrm{~T}$ & $31 \%$ & Bochum \\
$\mathrm{CD}_{2}$ & 0.25 & $\mathrm{CD}$ & $1 \mathrm{~K} / 6.55 \mathrm{~T}$ & $34 \%$ & Virginia \\
\hline
\end{tabular}

Table 3: Polarization results of deuterated polyethylene targets

factor $f$ compared to the deuterated alcohol materials, irradiated deuterated polyethylene can be an alternative as polarized deuteron target material under specific experimental conditions.

Finally, the radiation hardness of the polarization in scattering experiments is yet not measured. However, it is expected that those is reasonably good as for all radiation doped target materials.

\section{References}

[1] A. Abragam and M. Goldman, Rep. Progr. Phys., 41395 (1978).

[2] W. Meyer, Nucl. Instr. and Meth. A, 52612 (2004).

[3] St. Goertz, J. Harmsen, J. Heckmann, C. Hess, W. Meyer, E. Radtke, G. Reicherz, Nucl. Instr. and Meth. A, $\mathbf{5 2 6} 43$ (2004).

[4] St. Goertz, Habilitation thesis, Ruhr University Bochum, Germany,Bochum (2002) http://www.ep1.rub.de/poltarg/ptdata/theses/goertz_habil.pdf

[5] M. Iio, I. Daito, N. Doshita, T. Hasegawa, N. Horikawa, T. Inagaki, S. Ishii, T. Iwata, T. Kobayashi, et al., Nucl. Instr. and Meth. A, 526190 (2004).

[6] B. van den Brandt, E. I. Bunyatova, P. Hautle, J. A. Konter, S. Mango, Nucl. Instr. and Meth. A, 356 36 (1995).

[7] D. G. Crabb, Nucl. Instr. and Meth. A, 52656 (2004).

[8] D. G. Crabb, Proc.11th International Workshop on Polarized Sources and Targets, Tokyo 2005, p54.

[9] Li Wang, A. Berlin, N. Doshita, J. Herick, C. Hess, T. Iwata, K. Kondo, W. Meyer, G. Reicherz Nucl. Instr. and Meth. A 729, 36 (2013).

[10] S. Brown, et al., Proc. of the 4th Int. Workshop on Polarized Target Materials and Techniques, Germany,pp.66 1984.

[11] E. L. Cochran, F .J .Adrian, V .A .Bowers, J.Chem.Phys., 341161 (1961). 